\title{
Next Day Subjective and Objective Recovery Indices Following Acute Low and High Training Loads in Academy Rugby Union Players
}

\author{
Mark R. Noon ${ }^{1, *(\mathbb{D})}$, Rob S. James ${ }^{1}$ (D), Neil D. Clarke ${ }^{1}$, Richard J. Taylor ${ }^{2}$ and \\ C. Douglas Thake ${ }^{1}$ (iD) \\ 1 Faculty of Health and Life Sciences, Coventry University, Coventry CV1 5FB, UK; \\ apx214@coventry.ac.uk (R.S.J.); ab1633@coventry.ac.uk (N.D.C.); d.thake@coventry.ac.uk (C.D.T.) \\ 2 Department of Physical Education and Sports Studies, Newman University, Birmingham B32 3NT, UK; \\ rtaylor.runner@googlemail.com \\ * Correspondence: mark.noon@coventry.ac.uk; Tel.: +44-24-776-552-88
}

Received: 23 May 2018; Accepted: 10 June 2018; Published: 15 June 2018

\begin{abstract}
The aim of this study was to determine the sensitivity of selected subjective and objective monitoring assessments in detecting changes in group and individual responses to low and high load bouts of high intensity intermittent exercise. In a counterbalanced crossover design, Thirteen Academy Rugby Union players (mean \pm SD: age: $18 \pm 1$ years) performed a low load (15 min) and a high load (90 min) bout of high intensity intermittent exercise (Loughborough Intermittent Shuttle Test) one week apart. Monitoring assessments were performed immediately prior to and $20 \mathrm{~h}$ following each trial. Subjective self-report Well-being Questionnaire (WQ) items showed small to large deteriorations following the high load compared to low load ( $d=0.4-1.5, p=0.03-0.57)$. A very large increase in resting $\mathrm{HR}\left(\mathrm{HR}_{\text {rest }}\right)(d=2.1, p=0.02)$, moderate decrease in heart rate variability (HRV) indices $(d=0.7, p=0.04$ and $d=0.7, p=0.01$ for the natural logarithm of the standard deviation of R-R intervals (ln SDNN) and the root square of the mean squared differences of successive R-R intervals (rMSSD), respectively) and no change in countermovement jump $(d=0.0, p=0.97)$ were evident following the high load compared to low load. Individual WQ responses revealed 7/9, 7/9, $6 / 9,6 / 9,5 / 9,3 / 9$ and $1 / 9$ participants reported deteriorations in recovery, sleep quality, motivation, muscle soreness, fatigue, stress and appetite, respectively, following the high load compared to low load. Individual analysis indicated a negative response following the high load compared to low load in $\mathrm{HR}_{\text {rest }}$, ln SDNN and ln rMSSD for 4/6, 2/6 and 1/6 participants, respectively. Selected WQ items detected group and individual responses to high load and low load highlighting their potential utility. However, objective assessments lacked the sensitivity to detect small individual changes.
\end{abstract}

Keywords: athlete monitoring; athlete well-being; self-report questionnaire; performance

\section{Introduction}

Subjective and objective assessments applied to monitor an athlete's recovery status can assist coaches in effective training prescription, thus reducing the risk of non-functional overreaching (NFOR) alongside optimising the stimulus to promote training adaptation [1]. Accordingly, methods applied to monitor aspects of athlete recovery must be sensitive to changes in recovery status induced by different training loads. Self-reported well-being questionnaires have been proposed as valid measures to assess athlete responses to daily training and competition [2]. Previous studies have identified that daily self-report well-being questionnaires are sensitive to manipulations in training and competition load in high intensity intermittent team sport players [3-5]. The use of objective assessments to identify the 
maladaptive response associated with non-functional overreaching (NFOR) has received considerable attention [1,2]. Popular objective methods include countermovement jumps (CMJ) [1], resting HR $\left(\mathrm{HR}_{\text {rest }}\right)$ and heart rate variability (HRV) [6]. However, the sensitivity of these measures to assess athlete recovery remains equivocal with some studies reporting a sensitivity or lack of sensitivity to changes in training load [2,7].

Although sport teams often train together, as individuals the time-course of recovery from a physical training stimulus is dependent on numerous factors including initial level of fitness, genetics, prior recovery and training exposure [8]. Therefore it is important that training and recovery metrics are considered on an individual basis rather than just in relation to the training group. Hence, establishing an individual baseline, the error within the measure and what constitutes a meaningful change [9] is important to effective monitoring enabling practitioners to identify red flags and modify training for each individual [10]. Furthermore, the utility of subjective and objective assessments in a team sport setting is dependent on the assessment's ability to provide cost efficient, simple and accessible information on the athlete's time-course of recovery [11].

To date, no study has communicated how subjective and objective assessments respond on a group and individual basis to known high and low external training loads. Therefore, the aim of this study is to compare the sensitivity of selected subjective and objective monitoring assessments in detecting changes in group and individual responses to a low load and high load bout of high intensity intermittent exercise.

\section{Methods}

\subsection{Participants}

Thirteen Rugby Union players from an academy team competing in the Association of Colleges Midland Elite League (mean \pm SD: age $18 \pm 1$ years, stature $179 \pm 6 \mathrm{~cm}$, body mass $81.6 \pm 18.6 \mathrm{~kg}$ ) volunteered and provided informed consent for the study. Any participant who reported an injury during the study was excluded from any analysis. The study was undertaken during an in-season period. The participants' normal weekly training involved three to four pitch based Rugby sessions (120 min per session), 1-2 gym based sessions (45 min per session) plus an 80 min competitive match. The study was approved by an institutional Ethics Committee (P20975) and conformed to the declaration of Helsinki.

\subsection{Study Design}

Three weeks prior to the commencement of the study, reliability of the WQ, CMJ, and HR indices was established. All subjective and objective assessments were made on three occasions at 9.00 a.m., during a five day period during a week where no training was undertaken and there was no competitive match $(n=13)$. Participants did not take part in any structured recovery methods during this period.

Using a counterbalanced crossover design, participants $(n=10$, mean \pm SD: age $18 \pm 1$ years, stature $180 \pm 7 \mathrm{~cm}$, body mass $86.6 \pm 18.5 \mathrm{~kg}$, estimated $\mathrm{VO}_{2}$ max $48 \pm 4 \mathrm{~mL} \cdot \mathrm{kg} \cdot \mathrm{bm}^{-1}$ ) were assigned to a low load and a high load trial one week apart. Training load was manipulated by altering the duration of the LIST with the participant performing the Loughborough Intermittent Shuttle Test (LIST) [12] for $15 \mathrm{~min}$ (low load) or $90 \mathrm{~min}$ (high load). On the day following each trial (20 h post), subjective and objective monitoring assessments were carried out. Monitoring assessments included subjective (well-being questionnaire (WQ)) [3] and objective (countermovement jumps (CMJ), resting $\mathrm{HR}\left(\mathrm{HR}_{\text {rest }}\right)$ and resting heart rate variability (HRV)) measures. The participants carried out one LIST familiarisation $(2 \times 15$ min blocks $)$ two weeks prior to the study. To ensure consistency participants were familiarised with all monitoring assessments on a minimum of four occasions prior to undertaking the study. All testing was undertaken in a familiar environment where regular training and testing took place. Participants did not take part in any training in the week prior to or the between the trials. 
In addition, participants did not take part in any structured recovery methods during this period. The LIST and the CMJ were performed in an indoor sports hall. Measures of HR were taken in the changing rooms. Each trial and subsequent monitoring assessment was carried out at the same time each day (Figure 1) to avoid circadian variation.

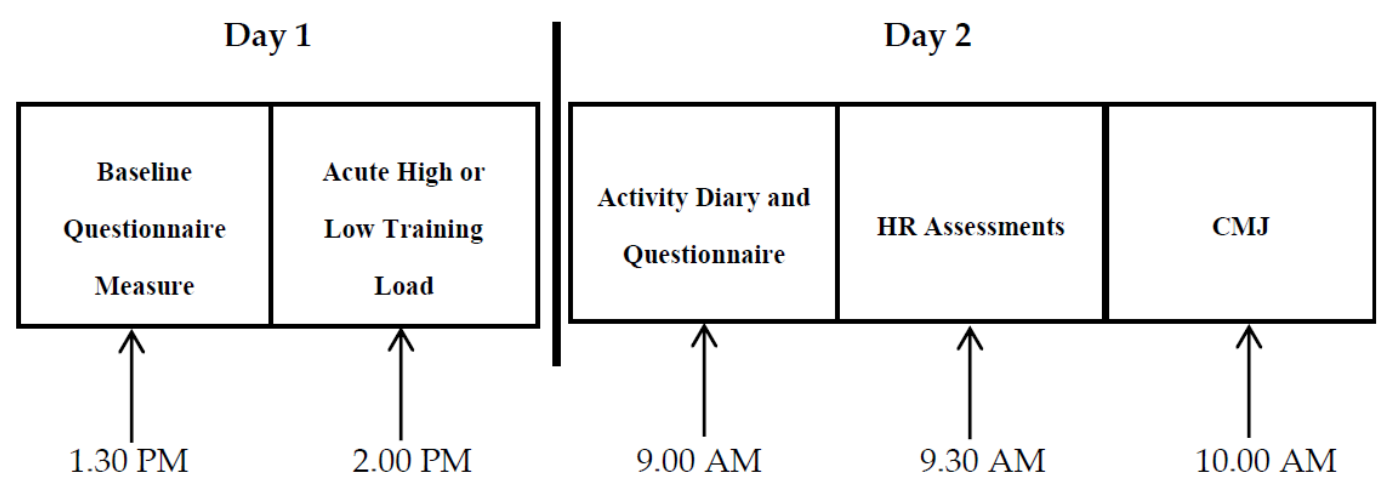

Figure 1. Schedule of each trial and subsequent objective and subjective assessments.

The study was conducted during low load training weeks where only one light session with a technical emphasis was completed and there were no competitive matches. In the seven days preceding each trial participants were asked to refrain from carrying out any of their own additional training. In an attempt to quantify any additional training participants were requested to complete an activity diary on a daily basis. Seven participants reported carrying out 1-3 additional upper body strength training sessions per week. These were at similar time points prior to the high load and low load trials. No other additional training was reported. Participants were asked to wear the same footwear on each day, carry out their normal breakfast regime and abstain from caffeine $12 \mathrm{~h}$ prior to attending testing sessions.

\subsection{Protocols}

\subsubsection{Loughborough Intermittent Shuttle Test (LIST)}

The LIST is a field based simulation designed to replicate the demands of intermittent team sports [12]. Participants were required to run at various speeds (sprinting, running, jogging and walking) determined by the group mean $\dot{\mathrm{VO}}_{2}$ max in an indoor sports hall (mean $\pm \mathrm{SD}$ : Temperature $17.4 \pm 0.4{ }^{\circ} \mathrm{C}$; Humidity $41.7 \pm 5.3 \%$ ). The group mean $\dot{\mathrm{VO}}_{2}$ max was estimated using the Yo-Yo intermittent recovery test level 1 [13] carried out two weeks prior to commencing trials. One block of the LIST (Part A) was completed (15 min) for the low load trial. Six blocks of the LIST (90 min), with a three minute intermission between each block, were completed for the high load trial. Following each block of the LIST each participant gave a Rate of Perceived Exertion (RPE) using the CR-10 scale [14]. Training load (AU) was calculated using Session RPE (RPE $\times$ duration).

\subsubsection{Well-Being Questionnaire (WQ)}

Questionnaires were completed immediately before and $20 \mathrm{~h}$ following each trial. Participants were asked to rate their perceptions of seven items each on a seven point scale (very good (+3), normal $(0)$ to very poor $(-3))$ to monitor their perceptions of well-being related to: motivation to train, quality of previous night's sleep, quality of recovery from previous day, appetite, feeling of fatigue, level of stress and level of muscle soreness [3]. Participants used a pen to complete a paper copy of the questionnaire on each occasion and did not discuss questionnaire responses with each other. For the purpose of analysis, the questionnaire items fatigue, stress and muscle soreness were reverse scored. Therefore, a higher score reflected greater fatigue, stress or muscle soreness. 


\subsubsection{Resting Heart Rate $\left(\mathrm{HR}_{\text {Rest }}\right)$ and Heart Rate Variability (HRV)}

HR measures were determined via similar methods to those previously described [15]. Participants were positioned in a supine position for $10 \mathrm{~min}$ in the changing rooms (mean $\pm \mathrm{SD}$ : Temperature $20.0 \pm 1.1^{\circ} \mathrm{C}$; Humidity $40.0 \pm 2.6 \%$ ). It was requested that the participants stayed as still as possible, refrained from talking and remained as relaxed as possible. No attempt was made to control breathing rate and depth. A heart rate monitor (Team System, Suunto, Vantaa, Finland) was worn by participants across the chest and measured HR beat to beat. The lowest HR in the final five minutes was used to determine $\mathrm{HR}_{\text {Rest }}$. In addition, the final five min was analysed using Kubios software version 2.1 (University of Eastern Finland, Koupio, Finland). Time domain measures of HRV, the natural logarithm of the standard deviation of R-R intervals (ln SDNN) and the root square of the mean squared differences of successive R-R intervals (ln rMSSD) were determined.

\subsubsection{Countermovement Jump (CMJ)}

All participants conducted a standardised 10 min warm up prior to the CMJ. The warm up involved a progressive increase in exercise intensity incorporating sport specific dynamic exercises (e.g., lunges, squats, kick throughs, skips, jumps) and running between two lines $20 \mathrm{~m}$ apart. CMJs were carried out in an indoor sports hall (mean \pm SD: Temperature $21.5 \pm 0.2{ }^{\circ} \mathrm{C}$; Humidity $47.5 \pm 2.4 \%$ ). Following a set of three warm up jumps, participants carried out a total of six unloaded CMJ. The jumps were carried out in two sets of three jumps interspersed with 2-3 min recovery between sets. Jump time was measured as flight via a contact mat (Fusion Sport, Canberra, Australia). There was a three to five second intermission between each of the three jumps in each set. The participant was instructed to attempt to jump as high as possible. The best jump was recorded. No information regarding jump technique was given. However jumps were disqualified if: either (1) a participant pulled their thighs up to their chest to extend their flight time; or (2) both feet did not land back on the jump mat. If a jump was disqualified, corrective feedback was given and the participant performed another jump. If corrective feedback was provided, a longer intermission of 15-20 s was required between jumps.

\subsection{Statistical Analysis}

Internal consistency of the WQ was assessed using Cronbach $\alpha$. Within-participant variation was expressed as typical error (TE) and calculated for CMJ and HR indices using the SD of the change in mean scores as described by Taylor, et al. [16]. TE was reported in absolute units and as a coefficient of variation $(\mathrm{CV})$. The smallest worthwhile change (SWC) was set as 0.2 of between participant standard deviation [9].

For group analysis the data was examined via the Wilk's Shapiro normality test. Paired $t$-tests were used to determine any differences between the low load and high load trials in normally distributed data. Non-normally distributed data including Session RPE and the subjective responses in the WQ were assessed using a bootstrapped paired $t$-test of 1000 replications [3]. Baseline WQ values prior to the low load and high load trials were compared. Pre to Post delta values from each trial were used to determine differences between WQ responses in the high load and low load trials. The uncertainty in the point estimate were reported as $95 \%$ confidence intervals. Effect sizes were reported using Cohen's $d$ (Trivial 0.00-0.19, small 0.20-0.59, moderate 0.60-1.19, large 1.20-1.99, very large >2.00) [17].

To determine individual responses to the high load and low load trials, the likelihood of a change for each individual was assessed considering the TE and the SWC. The likelihood of change is presented as percentage probabilities with a qualitative descriptor and any changes greater than $75 \%$ were considered substantial $[9,18]$. 


\section{Results}

\subsection{Reliability Data}

Internal consistency for the WQ ranged from $0.62-0.93 \alpha$ (Table 1 ). Reliability expressed as a coefficient of variation for objective assessments was $5.2 \%$ for CMJ, $6.0 \%$ for $\mathrm{HR}_{\text {rest }}, 4.9 \%$ for $\ln \mathrm{SDNN}$, $8.7 \%$ for rMSSD, (Table 1 ).

Table 1. Day to day reliability of well-being questionnaire (WQ) items, countermovement jump (CMJ) and heart rate (HR) indices.

\begin{tabular}{ccccccc}
\hline Item & Trial 1 Mean & Trial 2 Mean & Trial 3 Mean & Cronbach $\alpha$ & TE (CV) & SWC (CV) \\
\hline Motivation (AU) & $1.00 \pm 1.00$ & $1.00 \pm 1.22$ & $1.23 \pm 1.30$ & 0.78 & - & - \\
Sleep quality (AU) & $1.23 \pm 0.93$ & $1.15 \pm 1.41$ & $0.54 \pm 1.33$ & 0.71 & - & - \\
Recovery (AU) & $0.31 \pm 1.38$ & $1.08 \pm 1.19$ & $1.08 \pm 1.12$ & 0.62 & - & - \\
Appetite (AU) & $1.08 \pm 1.38$ & $1.23 \pm 1.17$ & $1.31 \pm 1.32$ & 0.82 & - & - \\
Fatigue (AU) & $-0.08 \pm 1.55$ & $-0.08 \pm 1.19$ & $-0.62 \pm 1.26$ & 0.65 & - & - \\
Stress (AU) & $-0.08 \pm 1.04$ & $0.15 \pm 1.72$ & $-0.08 \pm 1.44$ & 0.93 & - & - \\
Muscle soreness (AU) & $0.00 \pm 1.63$ & $-0.77 \pm 1.42$ & $-0.69 \pm 1.38$ & 0.77 & - & - \\
CMJ (cm) & $38.6 \pm 6.4$ & $39.0 \pm 5.5$ & $36.9 \pm 5.7$ & - & $2.0(5.2)$ & $1.5(3.9)$ \\
HR Rest (b·min ${ }^{-1}$ ) & $65 \pm 6$ & $68 \pm 7$ & $68 \pm 7$ & - & $4(6.0)$ & $2(3.0)$ \\
ln SDNN (ms) & $1.82 \pm 0.19$ & $1.84 \pm 0.15$ & $1.88 \pm 0.17$ & - & $0.09(4.9)$ & $0.04(2.2)$ \\
ln rMSSD (ms) & $1.73 \pm 0.29$ & $1.70 \pm 0.30$ & $1.76 \pm 0.30$ & - & $0.15(8.7)$ & $0.08(4.6)$ \\
\hline
\end{tabular}

Data expressed as mean \pm SD. Internal consistency of WQ items (motivation, sleep quality, recovery, appetite, fatigue stress and muscle soreness) assessed using Cronbach's $\alpha$. Typical error of measurement (TE) expressed as a coefficient of variation $(\mathrm{CV})$ is given for counter movement jump (CMJ), mean resting $\mathrm{HR}_{(\mathrm{HR}}$ Rest), the natural logarithm of the standard deviation of R-R intervals (ln SDNN) and the natural logarithm of the root square of the mean squared differences of successive R-R intervals (ln rMSSD). The SWC is also presented to allow comparison with typical variations $(n=13)$.

\subsection{Group Responses}

Session RPE was greater in the high load compared to low load (474 $\pm 187 \mathrm{AU}$, Table 2). Trivial to small differences were observed for items of the WQ between the two baseline measures $(d=0.1$ to 0.5 , $p=0.19-0.82$ ). Pre to post delta values were lower by a large extent for perceptions of sleep quality and of recovery following the high load compared to low load $(-1.0 \pm 1.1$ AU vs. $-0.3 \pm 1.1$ AU and $-2.4 \pm 1.8$ AU vs. $-0.2 \pm 1.7$ AU respectively, Table 2). Pre to post delta values for perceptions of motivation were moderately lower following the high load compared to low load $(-1.9 \pm 1.9$ AU vs. $-0.7 \pm 1.7 \mathrm{AU}$, Table 2). Pre to post delta values for perceptions of muscle soreness were moderately higher following the high load compared to low load (2.0 \pm 1.7 AU vs. $1.1 \pm 1.5$ AU, Table 2). Pre to post delta values were higher by a small extent for perceptions of appetite, fatigue and stress following the high load compared to low load (Table 2).

Table 2. Comparison between the effects of high load and low load trials on Session Rate of Perceived Exertion (RPE) during Loughborough Intermittent Shuttle Test (LIST) and subsequent WQ responses.

\begin{tabular}{cccccccc}
\hline & Low Load & $\begin{array}{c}\text { High } \\
\text { Load }\end{array}$ & Change & $\begin{array}{c}\text { Confidence } \\
\text { Interval }\end{array}$ & $p$ Value & Cohens $\boldsymbol{d}$ Effect Size \\
\hline Session RPE (AU) & $47 \pm 33$ & $521 \pm 174$ & $474 \pm 187$ & 369 to 579 & $<0.01$ & 3.8 & very large \\
Motivation (AU) & $-0.7 \pm 1.7$ & $-1.9 \pm 1.9$ & $-1.2 \pm 1.8$ & -2.2 to -0.1 & 0.12 & 0.7 & moderate \\
Sleep quality (AU) & $0.3 \pm 1.1$ & $-1.0 \pm 1.1$ & $-1.3 \pm 1.5$ & -2.3 to -0.6 & 0.12 & 1.2 & 1.5 \\
Recovery (AU) & $-0.2 \pm 1.7$ & $-2.4 \pm 1.8$ & $-2.2 \pm 2.4$ & -3.6 to -0.7 & 0.03 & large \\
Appetite (AU) & $0.0 \pm 1.7$ & $0.7 \pm 0.9$ & $0.7 \pm 2.1$ & -0.2 to 2.1 & 0.38 & 0.5 & small \\
Fatigue (AU) & $0.2 \pm 1.6$ & $0.9 \pm 1.6$ & $0.7 \pm 2.3$ & -0.8 to 1.9 & 0.41 & 0.4 & small \\
Stress (AU) & $0.2 \pm 0.2$ & $0.6 \pm 1.6$ & $0.3 \pm 1.7$ & -0.7 to 1.4 & 0.57 & 0.4 & small \\
Muscle soreness (AU) & $1.1 \pm 1.5$ & $2.0 \pm 1.7$ & $0.9 \pm 2.8$ & -0.8 to 2.6 & 0.36 & 0.6 & moderate \\
\hline
\end{tabular}

Mean \pm SD, 95\% confidence intervals, $p$ value, $\mathrm{t}$-statistic and effect size for Session RPE and pre to post trial delta values in both the high load and low load trials for perceptions of motivation, sleep quality, recovery, appetite, fatigue, stress and muscle soreness. Mean change \pm SD reported as a delta value from the high load to low load trial $(n=9)$. 
No differences in peak CMJ height were observed between high load and low load. A very large increase in mean $\mathrm{HR}_{\text {Rest }}$ was evident following the high load compared to low load $\left(6 \pm 4 \mathrm{~b} \cdot \mathrm{min}^{-1}\right.$, Table 3). Moderate decreases in indices of HRV were observed following the high load compared to low load $(-0.08 \pm 0.08 \mathrm{~ms}$, and $-0.13 \pm 0.08 \mathrm{~ms}$, for $\ln$ SDNN and ln rMSSD respectively, Table 3$)$.

Table 3. Peak CMJ performance and indices of HR at rest following the high load and low load trials.

\begin{tabular}{|c|c|c|c|c|}
\hline & Peak CMJ Height (cm) & Mean $H_{R} R_{\text {Rest }}\left(b \cdot \min ^{-1}\right)$ & ln SDNN (ms) & ln rMSSD (ms) \\
\hline Low Load & $37.2 \pm 4.4$ & $58 \pm 1$ & $1.96 \pm 0.09$ & $1.94 \pm 0.18$ \\
\hline High Load & $37.2 \pm 4.4$ & $64 \pm 4$ & $1.88 \pm 0.13$ & $1.81 \pm 0.18$ \\
\hline Mean Change & $0 \pm 1.8$ & $6 \pm 4$ & $-0.08 \pm 0.08$ & $-0.13 \pm 0.08$ \\
\hline Confidence Interval & -1.3 to 1.3 & 1 to 10 & -0.18 to 0.00 & -0.21 to -0.04 \\
\hline t-Statistic & -0.04 & 3.28 & -2.64 & -3.70 \\
\hline$p$ value & 0.97 & 0.02 & 0.04 & 0.01 \\
\hline Cohens $d$ & 0.0 & 2.1 & 0.7 & 0.7 \\
\hline Effect Size & trivial & very large & moderate & moderate \\
\hline
\end{tabular}

Mean \pm SD, 95\% confidence intervals, $p$ value, t-statistic and effect size for countermovement jump (CMJ) $(n=10)$ mean resting $\mathrm{HR}\left(\mathrm{HR}_{\mathrm{Rest}}\right)(n=6)$, the natural logarithm of: the standard deviation of R-R intervals $(\ln \mathrm{SDNN})(n=6)$ and the root square of the mean squared differences of successive R-R intervals (ln rMSSD) $(n=6)$.

\subsection{Individual Responses}

Training load, as indicated by Session RPE, ranged from 15 to 105 AU in low load compared to 240 to $810 \mathrm{AU}$ in high load (Table 4). The majority of participants showed poorer perceptions of well-being following the high load compared to low load trials; with 7/9,7/9,6/9,6/9, 5/9,3/9 and $1 / 9$ participants pre to post delta values for perceptions of recovery, sleep quality, motivation, muscle soreness, fatigue, stress and appetite deteriorating (Table 5). Only participant I reported WQ items that did not generally deteriorate following high load compared to low load. No participant had a substantial chance that the high load had a negative effect on CMJ performance compared to low load (5-72\%, Table 6). Four individual participants (A, B, F, G) had a substantially higher mean HRest following the high load compared to low load (76\% to $91 \%$, Table 7). Participants A and B yielded a substantially lower $\ln \mathrm{SDNN}$ following the high load compared to low load $(80 \%$ and $91 \%$, respectively Table 8). In addition, participant B showed a substantially lower $\ln$ rMSSD in high load compared to low load (82\%, Table 9). All other participants showed no substantial likelihood of change in HRV indices. Participants estimated $\mathrm{V}_{2}$ max values ranged from 39 to $52 \mathrm{~mL} \mathrm{~kg} \mathrm{~min}^{-1}$. Participants A, F and I had the largest and participant $\mathrm{G}$ had the smallest estimated $\mathrm{V}_{2}$ max values (Table 4).

Table 4. Estimated $\dot{\mathrm{VO}}_{2}$ max values and Session RPE during LIST in high load and low load trials for individual participants.

\begin{tabular}{cccc}
\hline \multirow{2}{*}{ Participant } & Estimated $\dot{V O}_{\mathbf{2}}$ Max $\left(\mathbf{m L ~ k g ~ b m}^{-\mathbf{1}}\right)$ & \multicolumn{2}{c}{ Session RPE (AU) } \\
\cline { 3 - 4 } & 52 & Low Load Trial & High Load Trial \\
\hline A & - & 60 & 420 \\
B & 50 & 75 & 405 \\
C & 48 & 15 & 525 \\
D & 49 & 75 & 240 \\
E & 51 & 15 & 750 \\
F & 39 & 15 & 585 \\
G & 47 & 60 & 810 \\
H & 51 & 30 & 600 \\
I & 45 & 15 & 375 \\
J & 57 & 105 & 495 \\
\hline
\end{tabular}

Data expressed as absolute individual scores for $\dot{\mathrm{VO}}_{2}$ max and Session $\operatorname{RPE}(n=10)$. 
Table 5. Individual differences in WQ responses between the high load and low load trials.

\begin{tabular}{cccccccc}
\hline Participant & Motivation & Sleep Quality & Recovery & Appetite & Fatigue & Stress & Muscle Soreness \\
\hline A & -2 & -1 & -3 & 0 & 0 & 0 & -1 \\
B & 2 & -1 & -1 & -1 & -4 & 0 & -4 \\
C & -2 & -1 & 0 & 0 & 3 & 4 & 1 \\
D & -3 & -2 & -4 & 0 & 2 & 0 & -1 \\
E & -3 & -5 & -6 & 1 & 4 & 1 & 6 \\
F & -2 & -1 & -1 & 0 & 1 & 0 & 2 \\
G & 1 & 0 & -3 & 6 & 0 & -1 & 1 \\
H & -2 & -1 & -4 & 0 & 1 & 1 & 3 \\
I & 0 & 0 & 2 & 0 & -1 & -2 & 1 \\
\hline
\end{tabular}

Perceptions of motivation, sleep quality, recovery, appetite, fatigue, stress and muscle soreness calculated as a pre to post trial delta value in both the high load and low load trials. Data presented as a change score between the high load and low load trial delta values $(n=9)$.

Table 6. Individual responses following high load and low load trials for peak jump height measured during a CMJ.

\begin{tabular}{|c|c|c|c|c|c|c|c|}
\hline \multirow{2}{*}{ Participant } & \multirow{2}{*}{ Low Load (cm) } & \multirow{2}{*}{ High Load (cm) } & \multirow{2}{*}{ Change (cm) } & \multicolumn{3}{|c|}{ Likelihood of Effect (\%) } & \multirow{2}{*}{ Qualitative Descriptor } \\
\hline & & & & -ve & Trivial & +ve & \\
\hline A & 40.3 & 38.6 & -1.7 & 53 & 34 & 13 & possibly, may not be -ve \\
\hline $\mathrm{B}$ & 36.0 & 32.8 & -3.2 & 72 & 22 & 5 & possibly, may not be -ve \\
\hline $\mathrm{C}$ & 32.3 & 33.4 & 1.1 & 18 & 37 & 44 & unlikely, probably not -ve \\
\hline $\mathrm{D}$ & 37.2 & 39.0 & 1.8 & 13 & 33 & 54 & unlikely, probably not -ve \\
\hline $\mathrm{E}$ & 44.7 & 43.9 & -0.8 & 40 & 38 & 21 & possibly, may not be - ve \\
\hline $\mathrm{F}$ & 41.5 & 41.0 & -0.5 & 36 & 39 & 24 & possibly, may not be -ve \\
\hline G & 30.5 & 31.0 & 0.5 & 24 & 39 & 26 & unlikely, probably not -ve \\
\hline $\mathrm{H}$ & 33.2 & 32.5 & -0.7 & 39 & 39 & 22 & possibly, may not be - ve \\
\hline I & 39.0 & 39.1 & 0.1 & 29 & 40 & 31 & possibly, may not be -ve \\
\hline $\mathrm{J}$ & 37.1 & 40.3 & 3.2 & 5 & 22 & 72 & unlikely, probably not -ve \\
\hline
\end{tabular}

Data presented as absolute scores, delta values, percentage of likelihood of change (negative ( - ve), trivial, positive $(+v e))$ and qualitative descriptor $(n=10)$. Smallest worthwhile change (SWC) and Typical Error (TE) from reliability data (Table 1) used to determine likely limits. $\mathrm{SWC}=1.5 \mathrm{~cm}$ and TE $=2.0 \mathrm{~cm}$.

Table 7. Individual responses following high load and low load trials for mean resting HR.

\begin{tabular}{|c|c|c|c|c|c|c|c|}
\hline \multirow{2}{*}{ Participant } & \multirow{2}{*}{$\begin{array}{l}\text { Low Load } \\
\left(b \cdot \min ^{-1}\right)\end{array}$} & \multirow{2}{*}{$\begin{array}{c}\text { High Load } \\
\left(b \cdot \min ^{-1}\right)\end{array}$} & \multirow{2}{*}{$\begin{array}{l}\text { Change } \\
\left(b \cdot \min ^{-1}\right)\end{array}$} & \multicolumn{3}{|c|}{ Likelihood of Effect (\%) } & \multirow{2}{*}{ Qualitative Descriptor } \\
\hline & & & & -ve & Trivial & +ve & \\
\hline $\mathrm{A}$ & 59 & 69 & 10 & 91 & 6 & 2 & likely, probably higher \\
\hline B & 57 & 66 & 9 & 89 & 8 & 3 & likely, probably higher \\
\hline C & 59 & 60 & 1 & 43 & 27 & 30 & possibly, may not be higher \\
\hline $\mathrm{F}$ & 57 & 65 & 8 & 85 & 10 & 4 & likely, probably higher \\
\hline G & 56 & 62 & 6 & 76 & 16 & 9 & likely, probably higher \\
\hline I & 59 & 59 & 0 & 36 & 27 & 36 & possibly, may not be higher \\
\hline
\end{tabular}

Data presented as absolute scores, delta values, percentage of likelihood of change (negative ( - ve), trivial, positive $(+\mathrm{ve})$ ) and qualitative descriptor $(n=6)$. Smallest worthwhile change (SWC) and Typical Error (TE) from reliability data (Table 1) used to determine likely limits. SWC $=2 \mathrm{~b} \cdot \mathrm{min}^{-1}$ and TE $=4 \mathrm{~b} \cdot \mathrm{min}^{-1}$.

Table 8. Individual responses following high load and low load trials for the natural logarithm of the standard deviation of normal to normal intervals (ln SDNN).

\begin{tabular}{cccccccc}
\hline \multirow{2}{*}{ Participant } & $\begin{array}{c}\text { Low Load } \\
(\mathbf{m s})\end{array}$ & $\begin{array}{c}\text { High } \\
\text { Load (ms) }\end{array}$ & $\begin{array}{c}\text { Change } \\
\text { (ms) }\end{array}$ & \multicolumn{2}{c}{ Likelihood of Effect (\%) } & Qualitative Descriptor \\
\cline { 5 - 6 } & & & $-\mathbf{v e}$ & Trivial & +ve & \\
\hline A & 1.88 & 1.66 & -0.22 & 91 & 6 & 3 & likely, probably lower \\
B & 2.00 & 1.85 & -0.15 & 80 & 12 & 7 & likely, probably lower \\
C & 1.91 & 1.89 & -0.02 & 44 & 24 & 32 & possibly, may not be lower \\
F & 1.88 & 1.84 & -0.04 & 50 & 23 & 27 & possibly, may not be lower \\
G & 2.02 & 1.99 & -0.03 & 47 & 24 & 29 & possibly, may not be lower \\
I & 2.09 & 2.03 & -0.06 & 56 & 22 & 22 & possibly, may not be lower \\
\hline
\end{tabular}

Data presented as absolute scores, delta values, percentage of likelihood of change (negative ( - ve), trivial, positive $(+\mathrm{ve}))$ and qualitative descriptor $(n=6)$. Smallest worthwhile change (SWC) and Typical Error (TE) from reliability data (Table 1) used to determine likely limits. $\mathrm{SWC}=0.04 \mathrm{~ms}$ and $\mathrm{TE}=0.09 \mathrm{~ms}$. 
Table 9. Individual responses following high load and low load trials for the natural logarithm of the root square of the mean squared differences of successive R-R intervals (ln rMSSD).

\begin{tabular}{|c|c|c|c|c|c|c|c|}
\hline \multirow{2}{*}{ Participant } & \multirow{2}{*}{ Low Load (ms) } & \multirow{2}{*}{ High Load (ms) } & \multirow{2}{*}{ Change (ms) } & \multicolumn{3}{|c|}{ Likelihood of Effect (\%) } & \multirow{2}{*}{ Qualitative Descriptor } \\
\hline & & & & - ve & Trivial & + ve & \\
\hline $\mathrm{A}$ & 1.66 & 1.51 & -0.15 & 63 & 23 & 14 & possibly, may not be lower \\
\hline $\mathrm{C}$ & 2.01 & 1.96 & -0.05 & 44 & 28 & 27 & possibly, may not be lower \\
\hline $\mathrm{F}$ & 1.79 & 1.73 & -0.06 & 46 & 28 & 26 & possibly, may not be lower \\
\hline G & 2.09 & 1.97 & -0.12 & 57 & 25 & 18 & possibly, may not be lower \\
\hline
\end{tabular}

Data presented as absolute scores, delta values, percentage of likelihood of change (negative ( - ve), trivial, positive $(+\mathrm{ve})$ ) and qualitative descriptor $(n=6)$. Smallest worthwhile change (SWC) and Typical Error (TE) from reliability data (Table 1) used to determine likely limits. SWC $=0.08 \mathrm{~ms}$ and TE $=0.15 \mathrm{~ms}$.

\section{Discussion}

The main finding of the present study were that group responses showed selected items of the WQ (motivation, recovery, sleep quality and muscle soreness), $\mathrm{HR}_{\text {rest }}$ and indices of $\mathrm{HRV}$ were sensitive to changes in training load. However, in the current study the CMJ was not sensitive to acute fluctuations in training load. Individual WQ responses revealed 7/9, 7/9, 6/9, 6/9,5/9,3/9 and 1/9 participants reported deteriorations in perceptions of recovery, sleep quality, motivation, muscle soreness, fatigue, stress and appetite, respectively following high load compared to low load. Also, 4/6, 2/6 and 1/6 individuals for $\mathrm{HR}_{\text {rest }}, \ln$ SDNN and $\ln \mathrm{rMSSD}$, respectively, reported a substantial chance of a negative response after high load compared to low load.

This study indicates that selected WQ items could provide important information on the recovery status of a player given their sensitivity to changes in high load and low load. Moderate to large deteriorations in perceptions of motivation, recovery, sleep quality and muscle soreness were evident following the high load compared to low load. Previously the WQ identified poorer perceptions of well-being in elite youth soccer players in the later seasonal training blocks, potentially due to an accumulation of training load [3]. In addition, other self-report questionnaires have shown sensitivity to changes in training load across in-season training weeks assessed using three questionnaire items (fatigue, sleep quality and muscles soreness) in elite soccer players [19]. Furthermore, Gastin, et al. [5] reported items of wellness (fatigue, muscle strain, hamstring strain, pain/stiffness, power, sleep quality, stress and well-being) improved on a daily basis throughout the week following a high competitive match load and subsequent lower loads throughout the week in Australian rules football players.

These previous studies provide an insight into the ecological validity of the use of self-report questionnaires in team sports. However, other stresses accumulated during these periods in addition to the training load reported such as additional training and non-sport specific stress may have contributed to the proposed dose-response relationship between perceptions of well-being and training load. In the present study, the high load and low load trials were carried out in a controlled low load training week. Therefore, the present study highlights the sensitivity of daily subjective self-report questionnaires to changes in recovery status induced by training load manipulations.

The WQ items fatigue, stress and appetite were not sensitive to the differing high load and low load trials. In contrast, a previous study reported fatigue and stress were sensitive to within training week variation in high load and low load [5]. One factor influencing these differences could be the magnitude of stress in the present study may have been lower given match simulations may not elicit physiological responses as high as for a competitive fixture [20]. Conversely, given the relative isolation of high load and low load in the present study, fatigue, stress and appetite may be sensitive to accumulated loads but not high acute loads.

The individual differences presented in this study highlight individual responses to a fixed bout of high intensity intermittent exercise. Participant I had the lowest RPE load and one of the highest estimated $\mathrm{VO}_{2}$ max values. Therefore, the relatively lower internal load could in part explain the lack of any changes in perceptions of well-being in the WQ for participant I. Conversely participants 
A and F reported poorer perceptions of well-being despite similar $\dot{\mathrm{V}}_{2}$ max values. This highlights confounding factors in addition to training load such as relationships and lifestyle [21] which could influence perceptions of well-being during recovery. Hence, it is important each athlete is assessed on an individual basis.

Subjective measures have been reported to show greater sensitivity to increased acute and chronic training loads in comparison with objective measures [2]. The present study reported group CMJ performance was not sensitive to changes in training load. However, $\mathrm{HR}_{\text {Rest }}$ and HRV were sensitive to changes in acute training loads.

CMJ is a simple assessment which could be used as an objective measure of neuromuscular performance prior to training (Twist and Highton 2013). However, the present study suggests that the CMJ measure using a contact mat is not sensitive to high training loads. In contrast, previous studies show decrements in CMJ performance $24 \mathrm{~h}$ and $48 \mathrm{~h}$ following a competitive fixture (Ascensao et al. 2011; Fatouros et al. 2010; Magalhaes et al. 2010) and a 90 min match simulation (LIST; Bailey et al. 2007; Magalhaes et al. 2010). These differences could reflect difference in the magnitude of the acute load (De Hoyo et al. 2016; Magalhaes et al. 2010). Furthermore, more expensive equipment such as force plates may be required to detect neuromuscular fatigue in a CMJ (Gathercole et al. 2015).

Group analysis of HR indices in the present study suggests $H_{R}$ Rest and HRV measures were sensitive to changes in training load. These measures of the autonomic nervous system have previously been proposed as a marker of NFOR and are reported to be sensitive to acute changes in training load [6,22]. Often team sport players are required to perform competitively twice a week [23]. Therefore, the sensitivity of HR indices to high acute training loads might be a useful tool for coaches and practitioners.

In an applied setting, monitoring must be carried out on an individual level due to the aforementioned individual differences. On an individual level it has been proposed that HR indices are too variable to assess athletes based upon a single measure (Buchheit 2014; Plews et al. 2013). Individual increases in $4 / 6$ participants were evident for $\mathrm{HR}_{\text {rest }}$, but only $2 / 6$ and $1 / 6$ participants reported a reduction in $\ln$ SDNN and $\ln$ rMSSD, respectively. Given the magnitude of the 'noise' and the SWC in measures of HR, single infrequent assessments of HR indices may only be sensitive to very large fluctuations in training load. Therefore, frequent daily assessment of HR indices using a rolling average would be required to reduce the 'noise' of the measurement which is often not practical in team sport players (Buchheit 2014; Plews et al. 2013).

A limitation to the present study was the small sample size. The study population was a convenience sample recruited from a group of rugby players competing at a select level. A follow-up study with more participants is needed to confirm the findings of the present study.

\section{Conclusions}

Subjective questionnaire items are sensitive to acute changes in training load and can be used as a standalone measure to assess the time-course of recovery in team sport players in an applied setting. Items such as recovery, sleep quality, motivation and muscle soreness were most sensitive to changes in training load and should be considered in the subjective self-report of athletes. CMJ were not sensitive to changes in acute training load, but HR indices showed sensitivity to changes in acute training load when assessed on a group level. However, when assessing athletes on an individual level, the large day to day variation in HR indices lacked the sensitivity to detect small meaningful changes. Given the time cost of data collection and analysis and the lack of sensitivity to individual changes, daily monitoring using objective assessments may not practical be in team sports. Therefore, daily subjective assessments may provide greater utility in an applied setting compared with objective assessments. 
Author Contributions: M.R.N. conceived the experiment. M.R.N., R.J.T., N.D.C., R.S.J., and C.D.T. designed and conducted the experiment. M.R.N. analyzed the data and performed statistical analysis. M.R.N. had primary responsibility for the final content. All authors have read and approved the manuscript.

Acknowledgments: The authors would like to thank: Tim Sparks for his advice on the statistical analysis; Matthew Ellis and Rhys Morris for their assistance with data collection and; the players and coaches for participating in the study.

Conflicts of Interest: The authors declare no conflicts of interest.

\section{References}

1. Halson, S.L. Monitoring training load to understand fatigue in athletes. Sports Med. 2014, 44 (Suppl. 2), S139-S147. [CrossRef] [PubMed]

2. Saw, A.E.; Main, L.C.; Gastin, P.B. Monitoring the athlete training response: Subjective self-reported measures trump commonly used objective measures: A systematic review. Br. J. Sports Med. 2016, 50, $281-291$. [CrossRef] [PubMed]

3. Noon, M.R.; James, R.S.; Clarke, N.D.; Akubat, I.; Thake, C.D. Perceptions of well-being and physical performance in English elite youth footballers across a season. J. Sports Sci. 2015, 33, 2106-2115. [CrossRef] [PubMed]

4. Thorpe, R.T.; Strudwick, A.J.; Buchheit, M.; Atkinson, G.; Drust, B.; Gregson, W. The Tracking of Morning Fatigue Status across In-Season Training Weeks in Elite Soccer Players. Int. J. Sports Physiol. Perform. 2016, 11, 947-952. [CrossRef] [PubMed]

5. Gastin, P.B.; Meyer, D.; Robinson, D. Perceptions of wellness to monitor adaptive responses to training and competition in elite Australian football. J. Strength Cond. Res. 2013, 27, 2518-2526. [CrossRef] [PubMed]

6. Buchheit, M. Monitoring training status with HR measures: Do all roads lead to Rome? Front. Physiol. 2014, 5, 73. [CrossRef] [PubMed]

7. Plews, D.J.; Laursen, P.B.; Stanley, J.; Kilding, A.E.; Buchheit, M. Training adaptation and heart rate variability in elite endurance athletes: Opening the door to effective monitoring. Sports Med. 2013, 43, 773-781. [CrossRef] [PubMed]

8. Faude, O.; Steffen, A.; Kellmann, M.; Meyer, T. The effect of short-term interval training during the competitive season on physical fitness and signs of fatigue: A crossover trial in high-level youth football players. Int. J. Sports Physiol. Perform. 2014, 9, 936-944. [CrossRef] [PubMed]

9. Hopkins, W.G. How to Interpret Changes in an Athletic Performance Test. Sportscience 2004, 8, 1-7.

10. Saw, A.E.; Kellmann, M.; Main, L.C.; Gastin, P.B. Athlete Self-Report Measures in Research and Practice: Considerations for the Discerning Reader and Fastidious Practitioner. Int. J. Sports Physiol. Perform. 2017, 12 (Suppl. 2), S2127-S2135. [CrossRef] [PubMed]

11. Saw, A.E.; Main, L.C.; Gastin, P.B. Role of a self-report measure in athlete preparation. J. Strength Cond. Res. 2015, 29, 685-691. [CrossRef] [PubMed]

12. Nicholas, C.W.; Nuttall, F.E.; Williams, C. The Loughborough Intermittent Shuttle Test: A field test that simulates the activity pattern of soccer. J. Sports Sci. 2000, 18, 97-104. [CrossRef] [PubMed]

13. Bangsbo, J.; Iaia, F.M.; Krustrup, P. The Yo-Yo intermittent recovery test: A useful tool for evaluation of physical performance in intermittent sports. Sports Med. 2008, 38, 37-51. [CrossRef] [PubMed]

14. Impellizzeri, F.M.; Rampinini, E.; Coutts, A.J.; Sassi, A.; Marcora, S.M. Use of RPE-based training load in soccer. Med. Sci. Sports Exerc. 2004, 36, 1042-1047. [CrossRef] [PubMed]

15. Manzi, V.; Castagna, C.; Padua, E.; Lombardo, M.; D’Ottavio, S.; Massaro, M.; Volterrani, M.; Iellamo, F. Dose-response relationship of autonomic nervous system responses to individualized training impulse in marathon runners. Am. J. Physiol. Heart Circ. Physiol. 2009, 296, H1733-H1740. [CrossRef] [PubMed]

16. Taylor, K.L.; Cronin, J.; Gill, N.D.; Chapman, D.W.; Sheppard, J. Sources of variability in iso-inertial jump assessments. Int. J. Sports Physiol. Perform. 2010, 5, 546-558. [PubMed]

17. Hopkins, W.G.; Marshall, S.W.; Batterham, A.M.; Hanin, J. Progressive statistics for studies in sports medicine and exercise science. Med. Sci. Sports Exerc. 2009, 41, 3-13. [CrossRef] [PubMed]

18. Al Haddad, H.; Simpson, B.M.; Buchheit, M. Monitoring changes in jump and sprint performance: Best or average values? Int. J. Sports Physiol. Perform. 2015, 10, 931-934. [CrossRef] [PubMed] 
19. Thorpe, R.T.; Strudwick, A.J.; Buchheit, M.; Atkinson, G.; Drust, B.; Gregson, W. Monitoring Fatigue During the In-Season Competitive Phase in Elite Soccer Players. Int. J. Sports Physiol. Perform. 2015, 10, 958-964. [CrossRef] [PubMed]

20. Magalhaes, J.; Rebelo, A.; Oliveira, E.; Silva, J.R.; Marques, F.; Ascensao, A. Impact of Loughborough Intermittent Shuttle Test versus soccer match on physiological, biochemical and neuromuscular parameters. Eur. J. Appl. Physiol. 2010, 108, 39-48. [CrossRef] [PubMed]

21. Meeusen, R.; Duclos, M.; Foster, C.; Fry, A.; Gleeson, M.; Nieman, D.; Raglin, J.; Rietjens, G.; Steinacker, J.; Urhausen, A.; et al. Prevention, diagnosis, and treatment of the overtraining syndrome: Joint consensus statement of the European College of Sport Science and the American College of Sports Medicine. Med. Sci. Sports Exerc. 2013, 45, 186-205. [CrossRef] [PubMed]

22. Bosquet, L.; Merkari, S.; Arvisais, D.; Aubert, A.E. Is heart rate a convenient tool to monitor over-reaching? A systematic review of the literature. Br. J. Sports Med. 2008, 42, 709-714. [CrossRef] [PubMed]

23. Carling, C.; Gregson, W.; McCall, A.; Moreira, A.; del Wong, P.; Bradley, P.S. Match running performance during fixture congestion in elite soccer: Research issues and future directions. Sports Med. 2015, 45, 605-613. [CrossRef] [PubMed]

(C) 2018 by the authors. Licensee MDPI, Basel, Switzerland. This article is an open access article distributed under the terms and conditions of the Creative Commons Attribution (CC BY) license (http://creativecommons.org/licenses/by/4.0/). 